Political Psychology, Vol. 36, No. 5, 2015

doi: 10.1111/pops.12171

\title{
MLK Day and Racial Attitudes: Liking the Group More but Its Members Less
}

\author{
William J. Chopik \\ University of Michigan \\ Ed O'Brien \\ University of Michigan \\ Sara H. Konrath \\ University of Michigan \\ University of Rochester Medical Center \\ Norbert Schwarz \\ University of Michigan
}

\begin{abstract}
Intuition suggests that the Martin Luther King holiday (MLK Day) should improve racial attitudes toward African Americans. However, its influence may depend on whether African Americans are evaluated as a group or individually. In two studies, we assessed racial attitudes either on MLK Day or on a control day. As might be expected, participants had more sympathetic attitudes towards African Americans as a group on MLK Day compared to control days; however, they evaluated individual African American exemplars more negatively on MLK Day compared to control days, who presumably seemed worse by comparison to the eminent political figure.
\end{abstract}

KEY WORDS: racial attitudes, evaluative judgment, contrast effects

What is the effect of Martin Luther King Day (MLK Day) on people's attitudes about African Americans? Perhaps thinking about Martin Luther King, Jr.- a prominent, successful, and highly respected African American figure-leads to more positive attitudes toward African Americans as a group. Recent research confirms that exposure to extremely positive African American exemplars, such as U.S. president Barack Obama, can improve attitudes towards African Americans (e.g., Columb \& Plant, 2011; Plant et al., 2009). Moreover, Martin Luther King, Jr. has specifically been shown to bypass a "White = American" stereotype (Rydell, Hamilton, \& Devos, 2010), suggesting that he could represent a unique prime within racial attitude paradigms. Might MLK Day improve attitudes toward African Americans by bringing attention to the prominent and well-respected African American political figure?

Perhaps. Yet, at the same time, there may be a more complex situation to consider. To our knowledge, the actual effects of MLK Day have never been scientifically investigated. However, 
related research on evaluative judgment suggests that there may be potential trade-offs associated with exposure to extremely positive exemplars like Martin Luther King, Jr. On the one hand, extremely positive exemplars have been shown to increase liking for the groups to which they belong. For example, encountering a strong agentic female confederate can improve opinions about women as leaders (Blair, Ma, \& Lenton, 2001), experience with a premium consumer product can improve opinions about the brand (Wänke, Bless, \& Igou, 2001), and exposure to a highly successful politician can improve opinions about his or her political party (Bless \& Schwarz, 1998). Such findings are known as assimilation effects, meaning that when an exemplar "fits" into that category, the positive attributes associated with this exemplar are extended to the group as a whole.

On the other hand, although positive exemplars can improve overall group perceptions, they have also been shown to undermine perceptions of specific group members. This is because such extreme positive examples invite comparisons that leave specific group members looking pale in comparison. For example, exposure to supermodels can make men rate their own wives as less attractive (Kenrick \& Gutierres, 1980), thinking about highly luxurious vacation destinations can diminish enjoyment for one's current trip (Raghunathan \& Irwin, 2001), and extremely successful politicians can make other specific politicians within the same party appear less desirable (Schwarz \& Bless, 1992a). Such findings are driven by contrast effects, by which the positive attributes associated with one specific exemplar are compared against other specific group members.

Thus, when considering whether MLK Day improves or harms attitudes towards African Americans, the above findings on evaluative judgment suggest a counterintuitive answer: MLK Day may both improve and harm racial attitudes depending on whether African Americans are judged as a group or individually. As reviewed above, when an exemplar (e.g., a successful female CEO) can be included within a general group category (e.g., women), assimilation effects occur (e.g., women as a group are rated as more competent). Contrast effects occur when this same exemplar (e.g., the $\mathrm{CEO}$ ) can be used to construct standards to which other specific group members are compared (e.g., other women are rated as less competent: Schwarz \& Bless, 1992a).

By this logic, Martin Luther King, Jr. can be included within representations of African Americans as a group (a superordinate category of which he is a member), but he can only be compared to (and not "included in") other individual African American political figures (lateral categories that are mutually exclusive). Hence, MLK Day should improve attitudes toward African Americans as a group (in line with related research by Rydell et al., 2010), but it could actually harm attitudes toward individual African American politicians, who likely fall short of his exceptional standard.

Such asymmetries in the impact of exemplars on group versus individual judgments have been reported in several domains. Importantly, however, evidence for their operation under naturalistic conditions is missing. Such evidence is needed to afford insight into real-world consequences related to how racial attitudes take shape outside the laboratory.

\section{The Present Research}

We used quasi-experimental designs in which college-student participants were randomly assigned to evaluate African Americans as a group and a specific African American exemplar, either on MLK Day or on a control day. However, because the effects of Martin Luther King, Jr. on racial attitudes should only occur if and when people are actually thinking about him, we also took into account whether participants attended or did not attend an on-campus MLK Day event. This was included because comparison-based contrast effects are limited to judgments for which the prime can serve as a highly accessible and relevant standard. Thus, our predictions should only pertain to people for whom Martin Luther King, Jr. is accessible at the time of judgment (Mussweiler, 2003). 
In other words, using the naturalistic holiday as a means to manipulate racial attitudes should only "work" to the extent that people are actually aware of its occurrence.

We expected that participants for whom MLK was salient (i.e., those attending an MLK Day event on the holiday) would have more sympathetic attitudes towards African Americans as a group but simultaneously more negative evaluations of a specific African American leader. Because this contrast should apply only to relevant or "matching" comparison targets that invite contrast (i.e., favorably viewed African American political leaders), we further predicted that judgments of less relevant targets would be unaffected by the holiday-lending further support to our proposed theoretical framework.

\section{Study 1}

\section{Method}

Participants. Randomly selected students $(N=1,366)$ at a Midwestern university were emailed to complete an online survey about "current issues." The final sample $(N=199)$ was $37.2 \%$ male aged 18-46 $(M=22.78), 80.4 \%$ Caucasian, 7.5\% Asian-American, 1.5\% African American, and 10.6\% Mixed or Other Ethnicity.

Procedure. Participants were randomly assigned to receive the survey invitation before (mode $=$ January $9 ; n=81$ ), on (mode $=$ January $19 ; n=66$ ), or after $($ mode $=$ February $2 ; n=52$ ) MLK Day 2004. The survey included an unrelated control question about whether the economy would get worse or better $(1=a$ lot better, $5=$ stay the same, $9=$ a lot worse $)$. Participants were asked a valid, widely used question assessing attitudes toward African Americans as a group: whether they thought discrimination against African Americans was still a problem $(1=$ no longer a problem, 9 = the single most significant problem; McConahay, 1986). We used this item because it measures indirect manifestations of racism with low social-desirability costs, bypassing traditional biases on explicit self-reports of anti-African American prejudice (McConahay, 1986). Participants additionally reported their own political orientation $(1=$ Democrat, $2=$ Moderate Democrat, 3 = Moderate Republican, $4=$ Republican).

Participants also reported their feelings toward a specific African American exemplar: Colin Powell $(1=$ dislike him a lot, $5=$ neutral, $9=$ like him a lot $)$. At the time of this study, Colin Powell was arguably the most widely recognized African American politician, thus serving as a politically relevant figure comparable to Martin Luther King. Indeed, favorability ratings for Colin Powell in 2004 were extremely high ( $87 \%$ favorability), and he was considered one of the most popular members of the presidential administration at the time (Gallup Poll, November 19-21, 2004). As a manipulation check, participants were asked at the end of the survey to report Colin Powell's race.

Participants also rated specific noncategorical exemplars in order to demonstrate that MLK Day should not affect judgments of unrelated targets. Jesse Jackson, a disliked African American politician at the time (38\% favorability; Gallup Poll, June 27-29, 2003) was included because disliked exemplars should not be as readily categorized into superordinate groups (Richeson \& Trawalter, 2005). We also included other well-known public individuals of the time, both African American and Caucasian American, to serve as control comparisons: Oprah Winfrey, Halle Berry, Denzel Washington, Bob Dole, David Letterman, Julia Roberts, and Tom Cruise. These exemplars were included to confirm that the predicted effects should be specific to positive African American political figures and not to general public figures who do not fit into the positive/African American/politician social category (Bless \& Schwarz, 1998).

Finally, following demographic questions, participants completing the survey before MLK Day were asked the question, "Are you planning on attending any events for the upcoming Martin Luther King Day celebrations?" ( $\left.n_{\text {yes }}=33 ; n_{\mathrm{no}}=41\right)$. Participants completing the survey on MLK Day were 
asked the question, "Are you planning on attending any events during the Martin Luther King Day celebrations? Or have you already attended any events?" $\left(n_{\mathrm{yes}}=16 ; n_{\mathrm{no}}=46\right)$. Participants completing the survey after MLK Day were asked the question, "Did you attend any events for the recent Martin Luther King Day celebrations?" ( $\left.n_{\text {yes }}=9 ; n_{\mathrm{no}}=38\right)$.

\section{Results and Discussion}

The two control days (before and after MLK Day) were collapsed into one control group (not MLK Day) because ratings of African Americans $(p=.90)$ and Colin Powell $(p=.99)$ did not differ between them.

Because separate groups of participants were asked about MLK Day event attendance on either MLK Day or a control day, we conducted a 2 (Date: MLK Day versus not) $\times 2$ (Holiday Accessibility: High versus Low) MANOVA on Target Evaluation Level (Group versus Individual). There was a significant three-way interaction between Target, Date, and Holiday accessibility, $F(1,179)=9.94, p=.002$ (see Figure 1). Decomposing this three-way interaction, the two-way interaction between Date and Target Evaluation Level was significant for participants who planned to attend an MLK Day event, $F(1,56)=12.80, p=.001$, as expected. Participants who attended or planned to attend an MLK Day event reported more sympathetic attitudes towards African Americans as a group on MLK Day $(M=6.88, S D=1.31)$ than otherwise $(M=5.33, S D=1.52), p=.002$, $d=1.07$. Conversely, these same participants rated Colin Powell more negatively on MLK Day $(M=5.19, S D=1.94)$ than otherwise $(M=6.57, S D=1.76), p=.01, d=.78$. As predicted, the two-way interaction was not significant for participants who did not [plan to] attend an MLK Day event, $F(1,123)=.25, p=.62$. Also as predicted, this interaction was not observed for ratings of White politicians $(p=.16)$, White entertainers $(p=.42)$, Black entertainers $(p=.99)$, or Jesse Jackson $(p=.87)$. This confirms that the negative consequence of the holiday is specific to targets who are highly relevant to the exemplar (i.e., Colin Powell versus Martin Luther King). Also, as expected, economic perceptions were unaffected by MLK Day, $p \mathrm{~s}>.40$, further suggesting that the influence of the holiday is specific to race-related items and not that it caused more general changes in evaluative judgments.

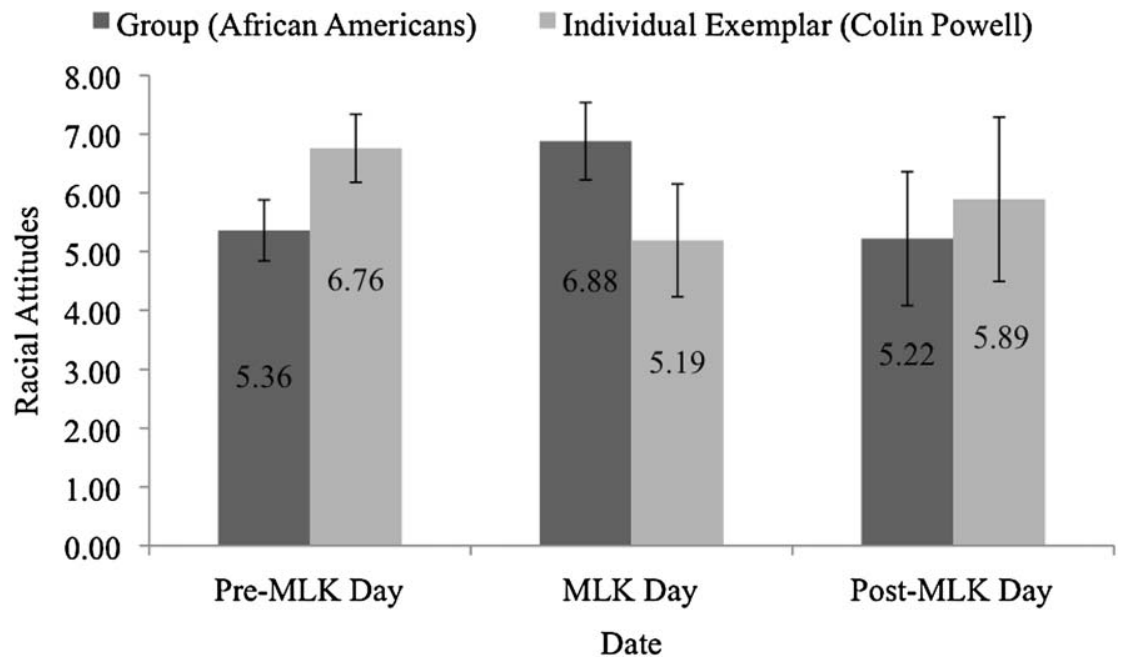

Figure 1. Interaction between target and date for the racial attitudes of participants who attended or planned to attend an MLK Day event (means) in Study 1. Error bars represent \pm 2 standard error. 
Due to the quasi-experimental nature of the current study, we took steps to ensure that our results were not attributable to other confounding variables. Most notably, people who participate in MLK Day events could be qualitatively different than those who choose not to attend them, which may have explanatory power over and above our predicted framework. To address this possibility, we conducted a binary logistic regression predicting event attendance (yes or no) from perceptions of changes in the economy, age, gender, race, and political orientation. None of these possible confounding variables predicted event attendance, all $p s>.25$. Further, in the MANOVA reported above, there was neither a main effect of event attendance on target rating nor an interaction between attendance and target ratings. In other words, individuals who attended an event on MLK Day did not give more positive ratings towards African Americans or Colin Powell overall-a prediction that would be made if these individuals were indeed different than those who chose not to attend an MLK event. It should also be noted that the university in this study requires MLK Day event attendance for many of its classes, clubs, and organizations, helping to explain this result-and further reduces chances of selection bias into one or the other condition.

\section{Study 2}

We distributed a similar survey during MLK Day 2012 in order to help rule out alternative hypotheses and address methodological limitations from Study 1. We included additional exemplars and control groups and updated the self-report measures to include multiple-item assessments of group attitudes. Barack Obama was chosen as the positive exemplar. Barack Obama (a Democrat) allows us to rule out that any ratings can be attributed to political ideology (Colin Powell was a Republican) or to some generational idiosyncrasies from the early 2000s. As the control day in Study 1 occurred early during the beginning of Black History Month (February in the United States), we chose a later control day in Study 2 (March 5) to rule out the possibility that evaluations of African Americans and exemplars stemmed from the accessibility of other positive African American figures during this month. We also asked direct questions about Martin Luther King, Jr. accessibility to assess if attending an event serves as an appropriate measure of Martin Luther King, Jr. awareness.

\section{Method}

Participants. Randomly selected students $(N=3,969)$ at a Midwestern university were emailed to complete an online survey about "emotions, attitudes, and perceptions." The final sample $(N=213)$ was $32.9 \%$ male aged $18-50(M=23.31), 80.3 \%$ Caucasian, $9.4 \%$ Asian-American, $1.4 \%$ Hispanic or Latino, and $8.9 \%$ Mixed or Other Ethnicity.

Procedure. Participants were randomly assigned to receive the survey on (mode = January 16; $n=106$ ) or after (mode $=$ March 5; $n=107$ ) MLK Day 2012. Participants completed the full six-item scale of modern racism (McConahay, 1986), from which the single-item from Study 1 was drawn. Another sample item is "Blacks should not push themselves where they are not wanted" $(1=$ strongly disagree $; 5=$ strongly agree $; \alpha=.74)$. Sniderman, Piazza, Tetlock, and Kendriek (1991) have found that attitudes toward government assistance for African Americans depend on whether the recipient is a group (African Americans) or a specific individual (one unemployed African American person), suggesting that evaluations may depend on the target of a judgment (an individual or a group, rather than attitudinal measures like the modern racism scale). Thus, participants also rated their general feelings towards African Americans $(1=$ dislike them a lot, $5=$ neutral, $9=$ like them a lot ) to provide a more complete picture of attitudes towards African Americans. As in Study 1, we included other (less relevant) groups to serve as control comparisons: ratings of Republicans, Democrats, Independents, Caucasian Americans, Hispanic/Latino Americans, and Asian-Americans. 
Participants also reported their feelings toward a specific African American exemplar: Barack Obama ( 1 =dislike him a lot, 5=neutral, 9=like him a lot). We included other famous (but unrelated) individuals of the time as control comparisons: Lady Gaga, Bill Clinton, George W. Bush, Mitt Romney, Justin Timberlake, Katy Perry, Beyoncé Knowles, Will Smith, Mike Tyson, Eminem, John Lennon, Oprah Winfrey, Joe Biden, Jesse Jackson, and Newt Gingrich. Herman Cain (22\% favorability; Gallup Poll, August 29-September 11, 2011) served as an additional disliked African American political exemplar.

Participants completing the survey on MLK Day were asked the question, "Did you attend (or plan to attend) a Martin Luther King Day event today?" $\left(n_{\mathrm{yes}}=32 ; n_{\mathrm{no}}=74\right)$. Participants completing the survey after MLK Day were asked the question, "Did you attend a Martin Luther King Day event in January?" $\left(n_{\mathrm{yes}}=31 ; n_{\mathrm{no}}=76\right)$. Participants also indicated the degree to which they had been thinking about Martin Luther King that day $(1=$ not at all, $3=$ somewhat, $5=$ very often; to test whether attending an MLK Day event serves as an appropriate measure of accessibility) and how important they considered MLK Day to them $(1=$ not important at all, $3=$ neutral $/$ mixed, $5=$ very important). Participants also reported their own political orientation $(1=$ extremely liberal, $6=$ extremely conservative).

\section{Results and Discussion}

First, we assessed the appropriateness of using event attendance as a measure of accessibility by conducting a two-sample $t$-test between the accessibility of Martin Luther King, Jr. of individuals who attended/planned to attend an event versus those who did not. People who attended/planned to attend an MLK event indeed had higher accessibility $(M=2.27, S D=1.22)$ of Martin Luther King, Jr. than those who did not $(M=1.32, S D=.59), t(211)=7.63, p<.001, d=1.15$.

We conducted a 2 (Date: MLK Day versus not) $\times 2$ (Holiday Accessibility: High versus Low) MANOVA on Target Evaluation Level (Group Rating, versus Modern Racism, versus Individual). There was a significant three-way interaction between Target Evaluation Level, Date, and Holiday accessibility, $F(2,208)=15.02, p<.001$ (see Figure 2 ). Decomposing this three-way interaction, the two-way interaction between Date and Target Evaluation Level was significant for participants who

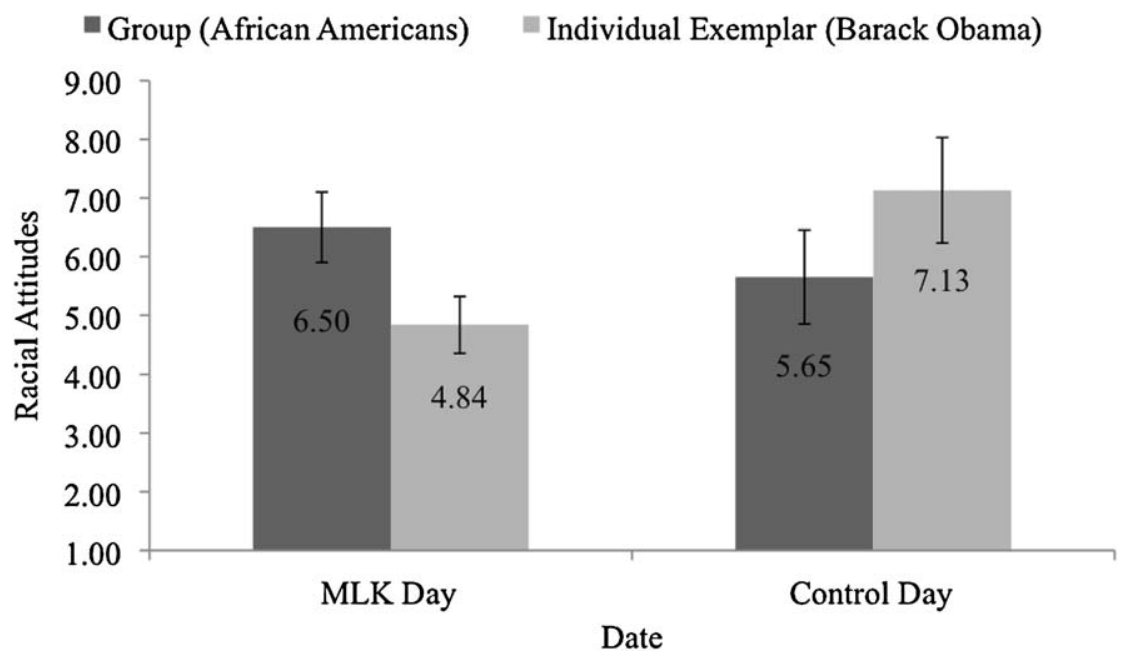

Figure 2. Interaction between target and date for the racial attitudes of participants who attended or planned to attend an MLK Day event (means) in Study 2. Error bars represent \pm 2 standard error. 
planned to attend an MLK Day event, $F(2,60)=11.84, p<.001$, as expected. Participants who attended or planned to attend an MLK Day event reported greater liking for African Americans as a group on MLK Day $(M=6.50, S D=1.70)$ than otherwise $(M=5.65, S D=1.36), p=.03, d=.56$. Consistent with Study 1, they also reported lower levels of modern racism on MLK Day $(M=1.50$, $S D=.43)$ than otherwise $(M=1.87, S D=.69), p=.01, d=.66$. Conversely, these same participants rated Barack Obama more negatively on MLK Day $(M=4.84, S D=2.27)$ than otherwise $(M=7.13$, $S D=2.51), p<.001, d=.97$. As predicted, the two-way interaction was not significant for participants who did not plan to attend an MLK Day event, $F(2,147)=.51, p=.61$. Also as predicted, this interaction was not observed for ratings of the other control groups: Republicans $(p=.49)$, Democrats $(p=.16)$, Independents $(p=.48)$, Caucasian Americans $(p=.13)$, Hispanic/Latino Americans $(p=.26)$, or Asian-Americans $(p=.20)$. This effect was further not seen for ratings of the other exemplars: White politicians $(p=.92)$, White entertainers $(p=.36)$, Black entertainers $(p=.67)$, Herman Cain $(p=.70)$, or Jesse Jackson $(p=.42)$.

Finally, we conducted a binary logistic regression predicting event attendance (yes or no) from age, gender, importance of MLK Day, political orientation, and race. Age was the only significant predictor of event attendance, $\beta=-.06, p=.02$, such that individuals who attended an MLK event were older $(M=24.73, S D=6.86)$ than those who did not $(M=22.71, S D=4.73)$. As in Study 1 , there was neither a main effect of event attendance on target ratings nor an interaction between attendance and target ratings. Thus, in the two studies, participants who attended an MLK event did not differ on several demographic or attitudinal characteristics that might be conceptually related to group and exemplar evaluations. However, participants who attended an MLK event reported higher accessibility of Martin Luther King, Jr. than those who did not, and only the predicted three-way interaction between Target, Date, and Holiday accessibility was significant.

\section{General Discussion}

Recent research finds that exposure to positive African American exemplars enhances perceptions of African Americans as a group (Columb \& Plant, 2011; Plant et al., 2009). However, the inclusion/exclusion model of evaluative judgment (Schwarz \& Bless, 1992a) predicts that prominent exemplars can improve perceptions of their group while simultaneously hurting perceptions of individual group members. This asymmetry exists because exemplars can be included within the representation of their group (a superordinate category of which they are a member) but not in the representation of another relevant exemplar (because lateral categories are mutually exclusive). Consistent with this prediction, we found that when Martin Luther King, Jr. was salient because of MLK Day, participants reported more sympathetic attitudes towards African Americans as a group but evaluated prominent African American politicians more negatively.

We extend earlier laboratory results (Schwarz \& Bless, 1992b) to naturalistic settings and highlight the potential dangers of comparing targets to idealized standards. Although intuition suggests that racial attitudes should not be susceptible to such minor situational influences, the patterns of change we observed in ratings for both African Americans and Powell/Obama fit well with existing theory and research suggesting that perceptions of groups and individuals are often the result of complex processes of assimilation and contrasts which yield a wide variety of outcomes (Corcoran \& Mussweiler, 2009).

Nonetheless, the degree to which racial attitudes are affected by the accessibility of relevant positive exemplars in everyday life may be more complex than the results observed here. In a recent study of implicit racial attitudes during Barack Obama's presidential campaign, for example, Schmidt and Nosek (2010) discovered that racial attitudes towards groups did not change much during the campaign and early presidency, even when examining significant moments in his campaign and media mentions of his name. Although this finding seems to conflict with the current study, 
there may have been a number of domain-specific differences. Although Obama's election marks a significant moment in race relations in the United States, his campaign and subsequent presidency was not necessarily involved in direct discussions of race-certainly not to the extent of Martin Luther King, Jr.'s civil rights movement during the 1960s, which constituted a major shift in public policy and national perception to a more egalitarian perspective. Therefore, the accessibility of Barack Obama may not influence racial attitudes to the same degree as a figure so long associated with racial equality and attitude change. Moreover, Schmidt and Nosek (2010) did not assess to what degree Obama was salient to individual participants, which may be necessary to change racial attitudes as our results suggest (see also Joy-Gaba \& Nosek, 2010).

On this note, a potential alternative explanation for our findings is that individuals who planned to attend an event on MLK Day may have viewed Martin Luther King, Jr. as more important, more self-relevant, or they may be more knowledgeable about race relations; in other words, our effects could be explained by a third variable because we did not randomly assign participants to attend an MLK Day event. Although possible, this would lead to a prediction of more positive racial attitudes overall and for specific exemplars, which is not what we found. We were also able to control for participants' self-rated importance of MLK Day. Moreover, in both studies, there was neither a main effect for attendance nor an interaction between attendance and target; only the predicted three-way interaction between attendance, target, and holiday date was observed. Attendance was also not explained by any participant demographic variables. Hence, any potential differences in perceived importance, self-relevance, or knowledge did not drive the observed effects. However, there remains the possibility that some unaccounted third variable may explain our results.

These findings provide many rich avenues for follow-up work. For example, future research should extend these findings to actual downstream behavior. Prior research demonstrates that priming specific exemplars, both explicitly and with more subtle cues, often affects motivation and effortful performance (Lockwood \& Kunda, 1997). In light of our findings, perhaps people cued with Martin Luther King, Jr. are more likely to actually participate in racially related events in February during Black History Month but less likely to attend a political rally or advocate for the views of a specific African American exemplar during that same time. Given the relatively low representation of high-ranking African Americans in contemporary politics, such decreased support may ironically hinder efforts for racial equity among elected officials during a holiday initiated to raise awareness of civil rights. Even if holidays like MLK Day have overall net positive effects on racial attitudes, such negative evaluations of specific African American politicians-individuals in perhaps the best position to initiate structural changes that facilitate more equal rights-is an important concern that warrants future investigation.

A final important extension of these findings might examine the potential effects of other prominent exemplars and whether our observed assimilation and contrast effects occur following random assignment. The assimilation and contrast effect from the current study also rely on the relatively small proportion of each sample that attended MLK Day events. Although we replicate our results in two studies at different points in time, future studies should randomly assign participants to high versus low accessibility of Martin Luther King, Jr. to examine the effects of this positive exemplar on ratings of African Americans and specific exemplars. Our exclusive use of college students is an additional limitation with regards to generalizing our results to the world at large. Although we find no evidence that college students should differ from the regular population in terms of such microprocesses examined in the current study (i.e., basic effects of accessibility on assimilation and contrast effects), future research should seek to include more representative samples.

Taken together, the present research highlights potential trade-offs of attempts to improve racial attitudes outside the laboratory, helping reveal novel insight into a critical real-world domain. 


\section{ACKNOWLEDGMENTS}

Correspondence concerning this article should be sent to William J. Chopik, Department of Psychology, University of Michigan, 530 Church Street, Ann Arbor, MI, 48104. E-mail: chopik@ umich.edu

\section{REFERENCES}

Blair, I. V., Ma, J. E., \& Lenton, A. P. (2001). Imagining stereotypes away: The moderation of implicit stereotypes through mental imagery. Journal of Personality and Social Psychology, 81(5), 828-841.

Bless, H., \& Schwarz, N. (1998). Context effects in political judgement: Assimilation and contrast as a function of categorization processes. European Journal of Social Psychology, 28(2), 159-172.

Columb, C., \& Plant, E. A. (2011). Revisiting the Obama Effect: Exposure to Obama reduces implicit prejudice. Journal of Experimental Social Psychology, 47(2), 499-501.

Corcoran, K., \& Mussweiler, T. (2009). Comparative thinking styles in group and person perception: One mechanism-Many effects. Social and Personality Psychology Compass, 3(3), 244-259.

Gallup Poll. (August 29-September 11, 2011). Poll questions retrieved from www.gallup.com.

Gallup Poll. (June 27-29, 2003). Poll questions retrieved from www.gallup.com.

Gallup Poll. (November 19-21, 2004). Poll questions retrieved from www.gallup.com.

Joy-Gaba, J. A., \& Nosek, B. A. (2010). The surprisingly limited malleability of implicit racial evaluations. Social Psychology, 41(3), 137-146.

Kenrick, D. T., \& Gutierres, S. E. (1980). Contrast effects and judgments of physical attractiveness: When beauty becomes a social problem. Journal of Personality and Social Psychology, 38(1), 131-140.

Lockwood, P., \& Kunda, Z. (1997). Superstars and me: Predicting the impact of role models on the self. Journal of Personality and Social Psychology, 73(1), 91-103.

McConahay, J. B. (1986). Modern racism, ambivalence, and the Modern Racism Scale. In J. F. Dovidio \& S. L. Gaertner (Eds.), Prejudice, discrimination, and racism (pp. 91-125). San Diego, CA: Academic Press.

Mussweiler, T. (2003). Comparison processes in social judgment: Mechanisms and consequences. Psychological Review, 110(3), 472-489.

Plant, E. A., Devine, P. G., Cox, W. T. L., Columb, C., Miller, S. L., Goplen, J., et al. (2009). The Obama effect: Decreasing implicit prejudice and stereotyping. Journal of Experimental Social Psychology, 45(4), 961-964.

Raghunathan, R., \& Irwin, J. R. (2001). Walking the hedonic product treadmill: Default contrast and mood-based assimilation in judgements of predicted happiness with a target product. Journal of Consumer Research, 28(3), 355-368.

Richeson, J. A., \& Trawalter, S. (2005). On the categorization of admired and disliked exemplars of admired and disliked racial groups. Journal of Personality and Social Psychology, 89(4), 517-530.

Rydell, R. J., Hamilton, D. L., \& Devos, T. (2010). Now they are American, now they are not: Valence as a determinant of the inclusion of African Americans in the American identity. Social Cognition, 28(2), 161-179.

Schmidt, K., \& Nosek, B. A. (2010). Implicit (and explicit) racial attitudes barely changed during Barack Obama's presidential campaign and early presidency. Journal of Experimental Social Psychology, 46(2), 308-314.

Schwarz, N., \& Bless, H. (1992a). Constructing reality and its alternatives: An inclusion/exclusion model of assimilation and contrast effects in social judgment. In L. L. Martin \& A. Tesser (Eds.), The construction of social judgments (pp. 217-245). Hillsdale, NJ: Lawrence Erlbaum.

Schwarz, N., \& Bless, H. (1992b). Scandals and the public's trust in politicians: Assimilation and contrast effects. Personality and Social Psychology Bulletin, 18(5), 574-579.

Sniderman, P. M., Piazza, T., Tetlock, P. E., \& Kendriek, A. (1991). The new racism. American Journal of Political Science, $35(2), 423-447$.

Wänke, M., Bless, H., \& Igou, E. R. (2001). Next to a star: Paling, shining, or both? Turning interexemplar contrast into interexemplar assimilation. Personality and Social Psychology Bulletin, 27(1), 14-29. 
